\title{
Swinging into thought: Directed movement guides insight in problem solving
}

\author{
LAURA E. ThOMAS \\ Vanderbilt University, Nashville, Tennessee \\ AND \\ Alejandro Lleras \\ University of Illinois, Urbana-Champaign, Illinois
}

\begin{abstract}
Can directed actions unconsciously influence higher order cognitive processing? We investigated how movement interventions affected participants' ability to solve a classic insight problem. The participants attempted to solve Maier's two-string problem while occasionally taking exercise breaks during which they moved their arms either in a manner related to the problem's solution (swing group) or in a manner inconsistent with the solution (stretch group). Although most of the participants were unaware of the relationship between their arm movement exercises and the problem-solving task, the participants who moved their arms in a manner that suggested the problem's solution were more likely to solve the problem than were those who moved their arms in other ways. Consistent with embodied theories of cognition, these findings show that actions influence thought and, furthermore, that we can implicitly guide people toward insight by directing their actions.
\end{abstract}

There is a growing commitment within cognitive psychology to understand the mind in the context of its ties to a physical body, championing the notion that the mind uses the body to accomplish cognitive goals, not only through direct action but also by tapping into perceptual and motor resources to represent and manipulate information (e.g., Barsalou, 1999; Glenberg, 1997; Wilson, 2002; Zwaan, 1999). These theories of embodied cognition suggest that cognitive processes are rooted in the body's interactions with the physical world; knowledge representation in the brain maintains properties of the sensorimotor states that give rise to it. Accumulating evidence shows that mental simulations, situated action, and bodily states underlie thought processes in perception, language comprehension, memory, social cognition, and conceptual processing (for a review, see Barsalou, 2008).

Although a wide variety of evidence points to strong interactions between the mind and the body, much of the research emphasis within embodied cognition has been tied to the notion that knowledge representations are based on body-bound mental simulations, essentially backing the claim that people use their bodies to think (e.g., Hegarty, 2004; Martin, 2007; Rubin, 2006). However, relatively little work has directly examined whether cognitive processes can arise from the manner in which our bodies interact with the immediate environment or whether it is possible to guide a person's thoughts by manipulating his or her actions. Are interactions between the mind and body bidirectional? The mind uses the body to accomplish cognitive goals, but can we make the body move in ways that guide the mind?
Research from the social psychology literature suggests that body movements may indeed play a causal role in cognitive processing, showing that experimental manipulations of actions and bodily states produce changes in affect. People are more likely to agree with statements that they hear if they nod while listening (e.g., Wells \& Petty, 1980 ), to rate a cartoon as more humorous if - outside of their explicit awareness - their smiling musculature is facilitated as they view the cartoon (Strack, Martin, \& Stepper, 1988), and to feel more pride in an achievement when directed to sit in an upright rather than slumped position when receiving news about performance (Stepper \& Strack, 1993). Moreover, actions with which people have extensive experience outside of laboratory manipulations can influence likeability judgments; for instance, people will find a random pair of letters more likeable if they have extensive typing experience and the letter pair is easy to type (Beilock \& Holt, 2007).

Some investigators have begun to examine how actions may also influence higher order cognition. Cook, Mitchell, and Goldin-Meadow (2008) found that children who were required to gesture while undergoing instruction on a new mathematical concept were more likely to retain the knowledge that they gained during instruction. Glenberg, Sato, and Cattaneo (2008) showed that participants' comprehension of both concrete and abstract language involving transfer was altered following $20 \mathrm{~min}$ of a repetitive transfer motor task. Thomas and Lleras (2007) demonstrated that by directing participants to move their eyes in a particular pattern, they could increase the participants' chances of solving

L. E. Thomas, laura.e.thomas@vanderbilt.edu 
a tricky problem. The same effect is also true with directed movements of covert attention (Thomas \& Lleras, 2009).

These findings hint at a causal link between action and cognition, but it is still unclear whether directed movements of the body can - outside of the participants' conscious awareness - guide higher order cognitive processing. We investigated the relationship between action and thought by asking participants to work their way through a classic insight problem ${ }^{1}$ while occasionally interrupting them with a seemingly unrelated exercise task. We evaluated whether the pattern of movement that the participants engaged in during exercise influenced their subsequent reasoning about how to solve the problem.

\section{The Problem}

The participants attempted to solve a variant of Maier's (1931) two-string problem pictured in Figures 1A and 1B. We asked the participants to find a way to tie two strings hanging from opposite ends of the room together using only their arms and the objects provided on a side table: a wrench, a paperback book, two small dumbbells, and a plate. Although the strings were long enough to be tied together, they were too short for the participants to be able to grasp the end of one string, walk to the opposite side of the room, and reach the other string. The most efficient way

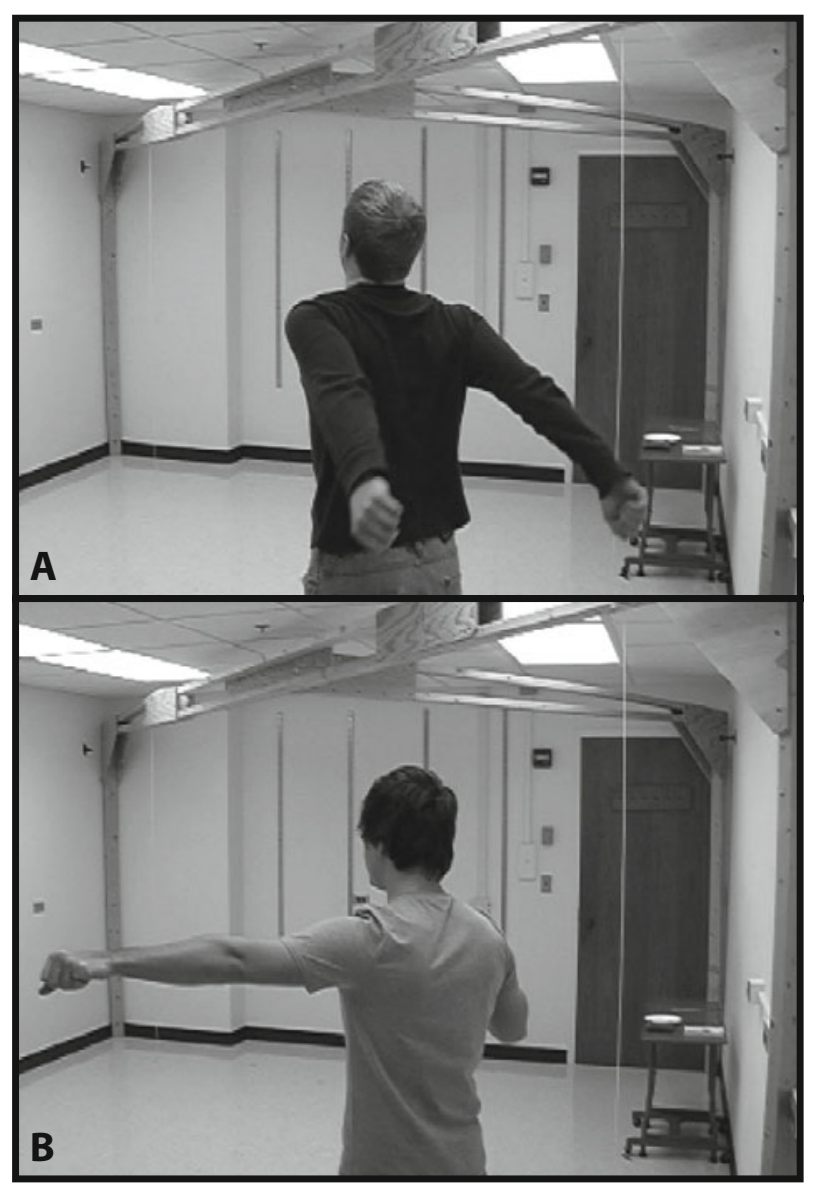

Figure 1. The two-string problem. (A) Swing condition. (B) Stretch condition. to solve this problem is to attach an object to one of the strings and use this object as a weight to make the string swing like a pendulum. Once one string is swinging, the participants can then walk to the stationary string, grasp it, and finally catch the moving string in its upswing.

To investigate how directed movements affect thought processes related to the two-string problem, we told the participants a cover story: that we were interested in how exercise breaks and blood oxygenation levels would influence their ability to solve the problem. We reasoned that if actions can guide cognitive processing, the participants directed to swing their arms during exercise (a movement consistent with the string motion necessary to solve the problem) should be more successful in solving the twostring problem than the participants directed to stretch their arms during exercise (a movement inconsistent with the problem's solution).

\section{METHOD}

\section{Participants}

Fifty-two ${ }^{2}$ University of Illinois students who were unfamiliar with the two-string problem participated for course credit.

\section{Procedure}

The problem space in which the participants worked is pictured in Figures $1 \mathrm{~A}$ and $1 \mathrm{~B}$. We asked the participants to tie the two strings together using only the objects provided on the table. The participants were not allowed to touch the strings with their legs or feet, to change the position of the strings on the wooden board to which they were attached, or to use the wooden board in any other way.

Following instruction on the two-string problem, the experimenter explained the cover story, telling the participants that we were interested in the influence of exercise breaks on problem-solving performance. The experimenter then silently demonstrated the movement that the participants were to perform during these exercise breaks. The participants were randomly assigned to one of two groups that differed in the type of motion their exercise required. The participants in the swing group extended their arms and swung them back and forth (see Figure 1A), whereas the participants in the stretch group alternated extending their arms straight out to either side in a stretching motion (see Figure 1B).

The experimenter told the participants that whenever they heard an alarm noise, they should move to an indicated position and begin performing the assigned movement until they heard a second alarm noise, at which time they were free to continue trying to solve the two-string problem until the next exercise break period. In addition, to prevent the participants from making any overt connections between directed movements and the problem-solving task, during the exercise periods, the participants also had to count backward by threes, aloud, beginning at a random number between 100 and 999 that the experimenter read at the start of each break period.

The experiment was divided into eight 2-min attempt intervals, with each interval consisting of a 20 -sec exercise period and a 100 -sec problem-solving period, during which the participants were free to move around the problem space, interact with the objects, and attempt solutions. ${ }^{3}$ If a participant tied the two strings together, he or she was scored as successful, the attempt number at which he or she solved the problem was recorded, and the experiment was concluded. The participants who were unable to tie the strings together after eight attempts (16 min) were scored as unsuccessful. All performances were recorded on a Panasonic 3CCD digital camcorder.

Following the end of the experiment, the participants completed a short posttest questionnaire. This questionnaire asked, "Did you see any relationship between the problem solving task and the exercise you performed? If yes, what was the relationship?" 


\section{RESULTS}

We were interested in whether the arm movements that the participants made during their exercise break periods influenced their chances of successfully solving the twostring problem. Figure 2 shows the proportion of the participants in each group to successfully solve the problem after each attempt interval. By the end of the 16-min deadline, $85 \%$ of the participants in the swing group had successfully solved the problem, whereas only $62 \%$ of the participants in the stretch group were successful. A log-rank survival analysis test (see Cleves, Gould, \& Gutierrez, 2002, for details) comparing the solution rates for the two groups across the eight 2-min intervals suggested that these rates were significantly different $\left[\chi^{2}(1,52)=3.95, p=.047\right]$.

In addition to demonstrating a causal link between directed action and cognitive processing, we also wanted to determine the extent to which this link is implicit. A check of the posttest questionnaires that the participants completed suggested that, as they were performing the task, only 3 participants suspected a relationship between their movements during exercise and the solution to the insight problem; 2 participants in the swing group and 1 participant in the stretch group wrote that they did see a relationship between their exercise and the problem's solution and then elaborated that they realized as they were trying to solve the problem that their directed arm movements were a hint about the solution. We reran the logrank survival analysis after excluding data from these 3 participants and found that the solution rates between the swing and stretch groups remained significantly different $\left[\chi^{2}(1,49)=4.30, p=.038\right]$.

\section{DISCUSSION}

Our results confirm the hypothesis that experimentally directed actions can influence thought in a problemsolving task: The participants who had to swing their arms

\section{Problem-Solving Success}

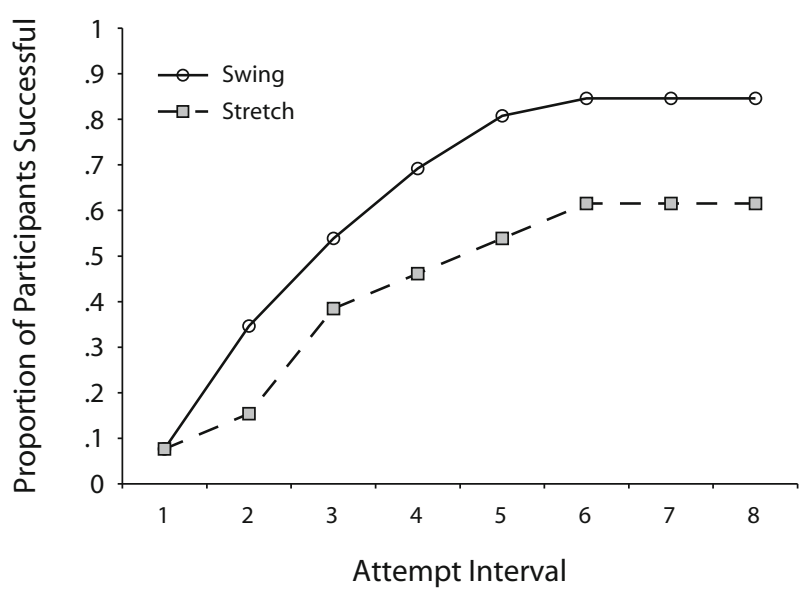

Figure 2. Proportion of the participants in each group to successfully solve the two-string problem after each attempt interval. in a pattern consistent with the two-string problem's solution were more likely to eventually solve the problem than were the participants who had to stretch their arms in a pattern that was not related to the problem's solution. The participants in the swing group tended to engage in behaviors that led them to more rapidly solve the twostring problem, frequently attaching an object to a string and setting it swinging after only a few repetitions of the arm-swinging exercise. The participants in the stretch group, however, were more likely to try unsuccessful methods of tying the strings together, often attempting to stretch and grab the second string while holding the first (to watch videos of the participants attempting to solve the problem, visit www.psych.uiuc.edu/ alleras/videos.htm). Furthermore, even though directed body movements had a significant impact on the participants' ultimate success or failure with the two-string problem, almost all of our participants reported being unaware of any relationship between how they moved their arms during exercise and how they went about trying to solve the problem. To the extent that our posttest questionnaire accurately captured how the participants perceived the relationship between their directed arm movements and the two-string problem, our results suggest that actions did implicitly guide thoughts.

The participants did not consciously perceive their swinging arm movements as overt hints about how to solve the two-string problem, presumably because the backward counting task that they engaged in during exercise tied up cognitive resources, preventing them from making explicit connections between their movements and the problem-solving task. This backward-counting task also likely prevented verbal rehearsal of words such as swing or stretch during exercise. However, we also replicated the results of this study in a second experiment, in which the participants did not have to count backward during exercise break periods. Under these conditions, the participants who swung their arms (78\% success rate) were also significantly more likely to solve the two-string problem than were the participants who stretched their arms $\left(50 \%\right.$ success rate) $\left[\chi^{2}(1,36)=4.55, p=.033\right]$, suggesting that actions influence thoughts regardless of the extent to which we attempt to make this influence implicit.

One question that arises from this research is whether the difference in performance between the swing and stretch groups was a result of the swinging movements facilitating thoughts about swinging the strings or of the stretching movements interfering with thoughts about swinging the strings. To address this question, we ran 18 participants in a separate control condition in which they were not asked to move during the break periods, nor to count backward: They were simply asked to stand still with their arms at their sides during those breaks. This group solved the twostring problem $72 \%$ of the time. Although this result may not be directly comparable with the main experimental conditions described here, the fact that these participants' performance fell in between the performances of the swing group (85\%) and the stretch group (62\%) suggests facilitation and interference driven specifically by our swinging and stretching movement interventions, respectively. 
Our results expand on a growing literature on embodied cognition in several interesting ways, demonstrating that embodiment goes beyond the mind's use of the body in accomplishing cognitive work (e.g., Barsalou, 2008; Wilson 2002). First, we showed that body movements can actually lead participants toward complex higher order thoughts that they would have been less likely to arrive at otherwise. Second, this effect occurs irrespective of whether the participants ever become aware of a relationship between the body movements and the problem at hand. And third, this effect takes place when we, the experimenters, are the ones asking the participants to move in specific ways; that is, we can literally change people's minds by asking them or leading them to move in a particular way.

Although previous studies of insight problem solving have shown that some participants unconsciously capitalize on hints to solve a problem, such as in Maier's (1931) original study, in which participants were more likely to produce the target solution after seeing the experimenter bump into a string to set it swinging, we believe the influence of a participant's actions on his or her thoughts in our experiment stands apart from these findings. In Maier's study, only the subset of the participants who tried and failed to produce the target solution to the two-string problem then saw the experimenter bump into a string. Of these participants, $62 \%$ eventually produced the target solution, in some cases only after viewing multiple string bumps and after being handed a pair of pliers and explicitly instructed that this item alone was sufficient to solve the problem. Because no baseline condition was measured (i.e., a no-hint group), interpretation of this "improvement" in success rate is difficult: It may have simply resulted from the overall longer exposure to the problem itself. In fact, in a more recent exploration of the two-string problem, the success rates of the participants who saw an experimenter bump into a string and set it swinging were not significantly different from those of a control group who did not receive any hints (Landrum, 1990). Even though these participants actually viewed the object that needed to move (the string) moving in the appropriate manner (swinging), only an average of 55\% of them solved the problem - a solution rate quite smaller than the ones we obtained in our swing groups. In other words, although it is possible that some sort of activation of the swinging concept may have been triggered in those studies, either by viewing the experimenter bump a string or by seeing the string itself swing, it seems that the action component in our experiments is substantially more potent in implicitly guiding thought than are those indirect hints. Furthermore, it is generally found that participants show weak or null effects of spontaneously transferring solution-related information from analogous problems to a new problem; participants often need explicit instructions in order to apply conceptual information from one instance to another (e.g., Gick \& Holyoak, 1980, 1983; Reed, Ernst, \& Banerji, 1974). In contrast, the embodied effects that we observed here were both strong and implicit, suggesting that actions influenced problem solving in a different way than did those indirect hints.
What possible mechanisms could account for this causal link between body and mind? Our results suggest that by getting a person to move, his or her threshold for experiencing thoughts that share something in common with the movement is lowered, but how is this possible? The interaction between body and mind may arise from the spatial compatibility that exists between different areas of the brain involved in representing spatial information (e.g., see Driver \& Spence, 2004, for multimodal compatibility, and Hommel, Müsseler, Aschersleben, \& Prinz, 2001, for perception-action compatibility). If we scale up from simple demonstrated cases of spatial compatibility - such as the fact that tactile stimulation on the same side as a visual target enhances activity in the visual cortex (Macaluso, Frith, \& Driver, 2000) - to more sophisticated interactions - such as activation of a swinging arm motion in the motor-control areas of the brain enhancing activity of representations of compatible object motion in other parts of the brain - we can see how certain actions may facilitate certain thoughts. The spatial representations used to direct a person's movements in space might be more active in motor-control areas of the brain, but because these representations are active, the person might be facilitated in representing compatible actions or locations in space in other parts of the brain. In the case of the two-string problem, preparing a swinging motion activates other swinging representations.

Alternatively, we can also conceptualize these compatibilities as interactions within the spatial module of working memory (e.g., Baddeley \& Hitch, 1974; Klauer \& Zhao, 2004; Logie, 1995). That is, if we assume that spatial working memory is invested both in the planning of movements and in reasoning about problems that are inherently spatial (as the two-string problem is), one can easily imagine that residual activation of location and action plans that a person uses to execute movements might interact with the spatial representations he or she uses to think about the spatial problem. Although the participants are consciously aware of the directed movements that they execute and the spatial representations of the two-string problem space, they do not necessarily have to be aware of the manner in which the former shape the latter. The contents of working memory are conscious, but the route by which they enter working memory need not be. In other words, participants need not necessarily be aware of residual activations relating to the exercise task, yet these activations could impact how (or which) spatial representations the participants use to attempt to solve the problem: Residual activation of the swinging arm action may make the participants working on the two-string problem more likely to include swinging motion of the strings in their spatial representation of that problem space.

\section{CONCLUSION}

By directing participants' arm movements, we were able to substantially affect their chances of successfully solving a demanding insight problem, demonstrating that specific movement interventions can implicitly guide higher order cognitive processing. This finding suggests that directed 
actions may be a powerful tool, not only in teaching us about the influence of movements on cognitive processes, but also as a potential intervention in learning situations (e.g., Cook et al., 2008). Our work also raises the possibility that self-generated actions could be instrumental in arriving at insights in a number of different domains of thought, such as scientific and creative thinking, and opens a new window into the unique nature of human cognition.

\section{AUTHOR NOTE}

The authors thank Diane Beck, David Irwin, Daniel Simons, and Ranxiao Frances Wang for their helpful input. Correspondence concerning this article should be addressed to L. E. Thomas, Psychology Department, 301 Wilson Hall, Vanderbilt University, 11121 st Avenue South, Nashville, TN 37203 (e-mail: laura.e.thomas@vanderbilt.edu).

\section{REFERENCES}

Baddeley, A. D., \& Hitch, G. (1974). Working memory. In G. H. Bower (Ed.), The psychology of learning and motivation: Advances in research and theory (Vol. 8, pp. 47-89). New York: Academic Press.

Barsalou, L. W. (1999). Perceptual symbol systems. Behavioral \& Brain Sciences, 22, 577-660. doi:10.1017/S0140525X99002149

Barsalou, L. W. (2008). Grounded cognition. Annual Review of Psychology, 59, 617-645. doi:10.1146/annurev.psych.59.103006.093639

BeILOCK, S. L., \& HolT, L. E. (2007). Embodied preference judgments: Can likeability be driven by the motor system? Psychological Science, 18, 51-57. doi:10.1111/j.1467-9280.2007.01848.x

Cleves, M. A., Gould, W. W., \& Gutierrez, R. G. (2002). An introduction to survival analysis using Stata. College Station, TX: Stata Press.

Cook, S. W., Mitchell, Z., \& Goldin-Meadow, S. (2008). Gesturing makes learning last. Cognition, 106, 1047-1058. doi:10.1016/ j.cognition.2007.04.010

DRIVER, J., \& SPENCE, C. (2004). Crossmodal spatial attention: Evidence from human performance. In C. Spence \& J. Driver (Eds.), Crossmodal space and crossmodal attention (pp. 179-220). Oxford: Oxford University Press.

Gick, M. L., \& HOLYOAK, K. J. (1980). Analogical problem solving. Cognitive Psychology, 12, 306-355. doi:10.1016/0010-0285(80)90013-4

Gick, M. L., \& HolyoaK, K. J. (1983). Schema induction and analogical transfer. Cognitive Psychology, 15, 1-38. doi:10.1016/0010 $-0285(83) 90002-6$

GLENBERG, A. M. (1997). What memory is for. Behavioral \& Brain Sciences, 20, 1-55. doi:10.1017/S0140525X97000010

Glenberg, A. M., SATo, M., \& CATTANeo, L. (2008). Use-induced motor plasticity affects the processing of abstract and concrete language. Current Biology, 18, R290-R291. doi:10.1016/j.cub.2008.02.036

Hegarty, M. (2004). Mechanical reasoning as mental simulation. Trends in Cognitive Sciences, 8, 280-285. doi:10.1016/j.tics.2004.04.001

Hommel, B., Müsseler, J., Aschersleben, G., \& Prinz, W. (2001). The theory of event coding (TEC): A framework for perception and action planning. Behavioral \& Brain Sciences, 24, 849-878.

Klauer, K. C., \& Zhao, Z. (2004). Double dissociations in visual and spatial short-term memory. Journal of Experimental Psychology: General, 133, 355-381. doi:10.1037/0096-3445.133.3.355

LANDRUM, R. E. (1990). Maier's (1931) two-string problem revisited: Evidence for spontaneous transfer. Psychological Reports, 67, 10791088. doi:10.2466/PR0.67.8.1079-1088

LogIE, R. H. (1995). Visuo-spatial working memory. Hove, U.K.: Erlbaum.

Macaluso, E., Frith, C. D., \& Driver, J. (2000). Modulation of human visual cortex by crossmodal spatial attention. Science, 289, 12061208. doi: $10.1126 /$ science. 289.5482 .1206
MAIER, N. R. F. (1931). Reasoning in humans: II. The solution of a problem and its appearance in consciousness. Journal of Comparative \& Physiological Psychology, 12, 181-194. doi:10.1037/h0071361

MarTin, A. (2007). The representation of object concepts in the brain. Annual Review of Psychology, 58, 25-45. doi:10.1146/annurev .psych.57.102904.190143

Metcalfe, J., \& Wiebe, D. (1987). Intuition in insight and noninsight problem solving. Memory \& Cognition, 15, 238-246.

ReEd, S. K., ERnST, G. W., \& BANERJI, R. (1974). The role of analogy in transfer between similar problem states. Cognitive Psychology, 6, 436-450. doi:10.1016/0010-0285(74)90020-6

Rubin, D. C. (2006). The basic-systems model of episodic memory. Perspectives on Psychological Science, 1, 277-311.

SMith, S. M., \& BlanKenship, S. E. (1991). Incubation and the persistence of fixation in problem solving. American Journal of Psychology, 104, 61-87. doi:10.2307/1422851

StepPer, S., \& Strack, F. (1993). Proprioceptive determinants of emotional and nonemotional feelings. Journal of Personality \& Social Psychology, 64, 211-220. doi:10.1037/0022-3514.64.2.211

Strack, F., Martin, L. L., \& StepPer, S. (1988). Inhibiting and facilitating conditions of the human smile: A nonobtrusive test of the facial feedback hypothesis. Journal of Personality \& Social Psychology, 54, 768-777. doi:10.1037/0022-3514.54.5.768

Thomas, L. E., \& Lleras, A. (2007). Moving eyes and moving thought: On the spatial compatibility between eye movements and cognition. Psychonomic Bulletin \& Review, 14, 663-668.

Thomas, L. E., \& Lleras, A. (2009). Covert shifts of attention function as an implicit aid to insight. Cognition, 111, 168-174. doi:10.1016/j .cognition.2009.01.005

Weisberg, R. W., \& Alba, J. W. (1981). An examination of the alleged role of "fixation" in the solution of several "insight" problems. Journal of Experimental Psychology: General, 110, 169-192. doi:10.1037/0096-3445.110.2.169

Wells, G. L., \& Petty, R. E. (1980). The effects of overt head movements on persuasion: Compatibility and incompatibility of responses. Basic \& Applied Social Psychology, 1, 219-230. doi:10.1207/ s15324834basp0103_2

Wilson, M. (2002). Six views of embodied cognition. Psychonomic Bulletin \& Review, 9, 625-636.

ZWAAN, R. A. (1999). Embodied cognition, perceptual symbols, and situation models. Discourse Processes, 28, 81-88.

\section{NOTES}

1. Insight problems are characterized by the fact that solutions seeming most obvious to naive problem solvers do not work, that problem solvers working on them cannot accurately track their own performance, and that problem solvers must often overcome an impasse in their reasoning in order to infer the problem's solution (Metcalfe \& Wiebe, 1987; Weisberg \& Alba, 1981).

2. Ten additional participants were excluded from the analysis for producing nonstandard solutions to the problem (such as lengthening one of the strings or pulling in a string with an object). Four belonged to the swing group, 6 to the stretch group.

3 . The intermittent nature of the exercise breaks leaves open the possibility that the participants experienced an incubation benefit (e.g., Smith \& Blankenship, 1991); that is, exercise breaks of either type may have helped the participants to let go of unsuccessful problem-solving strategies, clearing the way for them to potentially embrace the correct strategy in the next interval. However, since both the swing and stretch groups took exercise breaks at the same times and for the same durations, there is no reason to believe that such incubation effects would lead to differences in performance between the two groups.

(Manuscript received August 18, 2008; revision accepted for publication March 16, 2009.) 